\title{
Assessment of suicidal and self-injurious behaviours among patients with depression
}

\author{
A.A. Al-Habeeb, ${ }^{1}$ K.S. Sherra, ${ }^{2}$ A.M. Al-Sharqi ${ }^{3}$ and N.A. Qureshi ${ }^{7}$
}

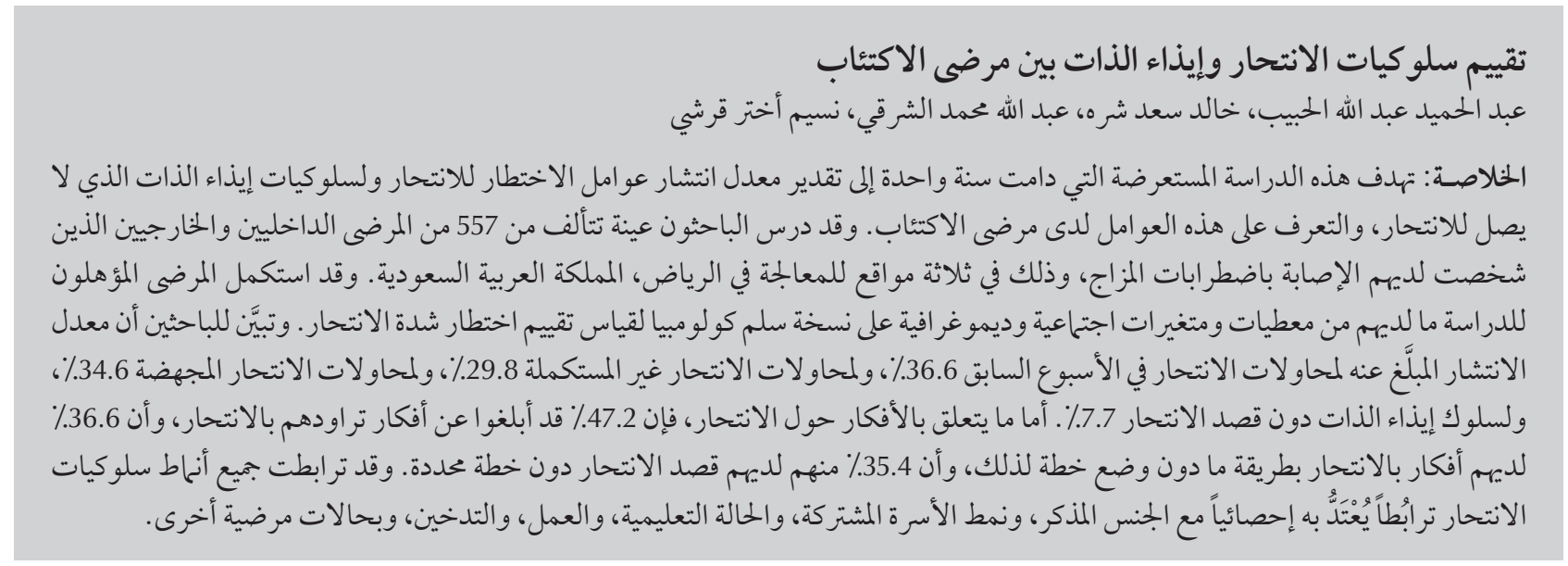

ABSTRACT The aim of this cross-sectional 1-year study was to estimate the prevalence and to identify the risk factors for suicidal and self-injurious behaviours among patients with depression. A convenience sample of 557 out- and inpatients diagnosed with mood disorders was selected from 3 treatment settings in Riyadh, Saudi Arabia. Eligible patients completed data on sociodemographic variables and the Columbia suicide severity rating scale risk assessment version. The reported prevalence of attempted suicide in the previous week was $36.6 \%$, interrupted suicide attempt $29.8 \%$, aborted suicide attempt $34.6 \%$ and self-injurious behaviour without suicide intent $7.7 \%$. Concerning suicide ideation, $47.2 \%$ reported suicidal thoughts, $36.6 \%$ suicidal thoughts with methods but without a specific plan and $35.4 \%$ suicidal intent without a specific plan. Male sex, joint family type, literate education, being in employment, smoking and physical co-morbidities were significantly associated with all types of suicide behaviour.

\section{Évaluation des comportements suicidaires et automutilatoires chez des patients atteints de dépression}

RÉSUMÉ La présente étude transversale sur un an visait à identifier les facteurs de risque des comportements suicidaires et automutilatoires chez des patients atteints de dépression et à estimer leur prévalence. Un échantillon de commodité de 557 patients consultant ou séjournant dans trois établissements de soins de Riyad (Arabie saoudite) après avoir reçu un diagnostic de troubles de l'humeur a été sélectionné. Les patients éligibles ont fourni des informations concernant des variables sociodémographiques puis ont rempli la version d'évaluation du risque de l'échelle Columbia de la gravité du risque de suicide (Columbia Suicide Severity Rating Scale). La prévalence rapportée au cours de la semaine précédente était de 36,6 \% pour les tentatives de suicide, de 29,8 \% pour les tentatives de suicide interrompues, de 34,6\% pour les tentatives de suicide avortées et de 7,7 \% pour les comportements automutilatoires sans tentative de suicide. Concernant l'idéation suicidaire, 47,2\% des patients ont rapporté avoir eu des pensés suicidaires, 36,6 \% des pensées suicidaires associées une méthode mais sans projet particulier, 35,4\% l'intention de se suicider sans projet précis. Être de sexe masculin, faire partie d'une famille élargie, être alphabétisé, avoir un emploi, consommer du tabac, souffrir de comorbidités physiques étaient des caractéristiques fortement associées à tous les types de comportement suicidaire.

${ }^{\prime}$ General Administration for Mental Health and Social Services, Ministry of Health, Riyadh, Saudi Arabia. ${ }^{2}$ Department of Psychiatry, Faculty of Medicine, University of Mansoura, Mansoura, Egypt. ${ }^{3}$ Specialized Psychiatry Clinics, Riyadh, Saudi Arabia (Correspondence to N.A. Qureshi: qureshinaseem@live.com).

Received: 03/04/11; accepted: 11/01/12 


\section{Introduction}

Suicide and non-suicide behaviours are major public health problems worldwide $[1-3]$. Around $20 \%$ to $60 \%$ of deaths by suicide occur among people who have a mood disorder [1]. For those with bipolar disorder the suicide risk is 15 times that of the general population [3]. Furthermore $50 \%$ of people who kill themselves have a history of deliberate self-harm with suicidal intent, an episode having occurred within the year before death in $20 \%-25 \%$ of cases [4-8]. About $10 \%$ of patients with deliberate self-harm behaviour ultimately die by suicide [9]. Yet it is believed that $25 \%-50 \%$ of those who contemplate or attempt suicide do not consult a health provider [10].

The identified key risk factors of suicidal behaviour among patients with depression include, among others, previous self-harm, severity and duration of the illness, alcohol or drug abuse, serious chronic physical illnesses, lack of a partner, comorbid anxiety and personality disorders, a family history of suicide and major stressful life events [11]. In a 5-year follow-up study of repeaters of suicidal attempts, it was reported that high anxiety, severe depression, psychiatric symptoms and young females with low education were significantly associated with repetition of attempted suicide [12]. Similarly, the identified protective factors against suicide and self-injurious behaviour are numerous, including treatment of underlying mental disorders, alcohol and drug abuse, physical disorders and others [13-18].

The literature on self-injurious behaviours, with and without intent to die, is scanty from Eastern Mediterranean countries. A retrospective study of 365 attempted suicides in Riyadh, Saudi Arabia, revealed that the most important motives for attempting suicide were family/marital problems and psychiatric disorders especially mood disorders. Females who attempted suicide were predominantly having family problems, whereas males were more often suffering from affective disorders [19]. This study aimed to estimate the prevalence and explore the risk and protective factors underpinning attempted suicide and other self-harm behaviours among patients with depression in Saudi Arabia. To our knowledge, there has been no other similar study in Saudi Arabia. The rationale of conducting this research was that self-harm behaviour of variable severity is commonplace and mostly coupled with psychiatric disorders especially depression. The expected findings including rate of self-harm behaviour and its underlying sociocultural factors may help in developing preventive strategies against self-harm behaviour in Saudi Arabia.

\section{Methods}

\section{Study setting and sample}

This study was conducted over a 1-year period from 1 November 2008 to 31 October 2009. A convenience sample of patients with diagnosed depression $(n=557)$ was selected from Al-Amal Complex for Mental Health in Riyadh $(n=250)$, Buraidah Psychiatric Hospital $(n=225)$ and Medina Psychiatric Hospital $(n=82)$. These hospitals were selected because assistant investigators from these 3 settings showed a keen interest to help collect data after detailed interview of the participants.

The sample was consecutive outand inpatients who met all the following inclusion criteria: (1) aged $\geq 16$ years and $<60$ years; (2) fulfilling the Diagnostic and Statistical Manual of Mental Disorders (4th edition) criteria of mood disorder, with or without intent to kill behaviours; (3) having mood disorders comorbid with other mental disorders; (4) with repeat selfharm with and without intention to die; and (5) with medical problems but not those causing psychosis or depression.
The number of patients above the age of 60 years attending general psychiatric hospitals is very low due to the lack of specialized services for the elderly population in Saudi Arabia. The mood disorders considered for this study were mainly depression, bipolar disorder and postpartum depression. The following were patients were excluded: (1) those with brain injuries; (2) with psychoses caused by medical and neurological conditions; and (3) with drug and alcohol abuse. Every 3rd patient was selected consecutively on 5 working days of the week. The total number of patients recruited in the 3 months was 557. The refusal rate was $10 \%$.

\section{Data collection}

Following a detailed interview with patients, their sociodemographic and clinical variables were abstracted on a semi-structured sheet and they were given the Columbia suicide severity rating scale (C-SSRS) risk assessment version. This scale has been used in clinical drug trials involving patients with depression and suicidal communications [20]. It has good reliability and validity and assesses actual attempts, non-suicidal self-injurious behaviour, interrupted attempts, aborted attempts, preparatory acts or behaviours, absence of suicidal behaviour and 5 types and frequency of suicidal ideations over the previous 1 week and also risk and protective factors of suicide.

One of the 3 bilingual interviewers, all psychiatrists, read the probes and definitions of each of the subitems of 6 domains of this scale slowly and clearly to the patient and the responses were noted by the interviewer on the template. The interviewers were invited twice to mini-workshops for detailed discussion and interpretation of this tool in both English and Arabic languages before starting the study. The questionnaire was delivered in English with explanations in Arabic language given patients who did not understand. 
The respondents were arbitrarily categorized into 4 income groups based on annul total income in Saudi riyals (SR): low $(S R \leq 25000)$, middle $(\mathrm{SR} \geq 25000-\leq 50000)$, high (SR $>50000$ to 99000$)$ and very high ( $\geq$ SR $100000)$.

The complete research proposal including informed consent form was submitted to the General Administration for Medical Research, Ministry of Health $(\mathrm{MOH})$ that has both scientific and ethics committees for evaluating submitted research protocols. Permission was obtained for conducting this research in $\mathrm{MOH}$ settings. All participants were requested to sign the consent form.

\section{Terminology}

The following terms were used in this research: suicide attempt (uncompleted suicide: intentional act of selfdestruction without fatal outcome); aborted suicide attempt (intentional act of self-destruction that was aborted due to internal mind-body defensive reactions); interrupted suicide attempt (intentional act of self-destruction that was interrupted by external human forces); suicidal ideation (cognitive thinking about suicide but without communicating to others or carrying out the thoughts into act); self-injurious behaviour (deliberate self-harm, non-suicidal self-injurious behaviour, self-mutilation and non-accidental selfinjurious behaviour).

\section{Data analysis}

The data was entered in the computer and cleaned and analysed by SPSS, version 10 . Besides calculating the frequency distribution and statistics of the variables, odds ratios (OR) were estimated by comparing binary socioclinical and risk factors of suicidal and self-injurious behaviours related to the 36 items except 2 clinical items related to major depressive and mixed affective episode of all 6 domains of the CSSRS risk assessment version. The last domain includes 6 items of protection against risk of suicide. Because of space constraints, we have reported only those results reflecting significant strength between 2 groups. A probability $P$-value of $\leq 0.05$ was considered significant.

\section{Results}

\section{Sociodemographic and clinical background}

The mean age of the 557 respondents was 39.0 (SD 11.7) years (range 16-60 years). A majority of participants were males (77.6\%), of Saudi Arabian nationality (94.3\%) and with primary to college education (68.6\%). More than half were unemployed (63.4\%) and $76.8 \%$ were married. About $51.7 \%$ of respondents were from joint families while $48.3 \%$ were living in nuclear families. A great majority were Muslims (99.3\%) and from the low- to middleincome categories (86.4\%). Two-thirds were from an urban area (65.7\%). A total of $338(60.71 \%)$ reported being smokers, all males.

Physical comorbidity was found among $24.8 \%$ of participants. Comorbidity with other psychiatric disorders was noted among $48.3 \%$ of participants. Major depression and mixed affective episodes were detected among $96.8 \%$ and $5.2 \%$ of participants respectively, while 7 patients (1.3\%) had postpartum depression. The frequency of other items from the C-SSRS (activating events, treatment history and clinical status) are shown on Table 1.

\section{Actual suicide, suicide ideation and self -injurious behaviour}

Out of the total sample of depressed patients $36.6 \%$ reported actual attempted suicide in the previous week, $29.8 \%$ interrupted suicide attempt and 34.6\% aborted or self-interrupted suicide attempt. Other preparatory acts to kill self were reported by $18.9 \%$ of respondents and self-injurious behaviour without suicide intent by $7.7 \%$ (Table 2). Most of the respondents reported that they had minor injuries $(16.9 \%)$ or very minor/no injuries (51.7\%) as a result of suicide attempts. Furthermore a majority admitted to "behaviour not likely to result in injury" (31.6\%) and "behaviour likely to result in injury but not likely to cause death" (39.0\%).

The frequency of items of suicidal ideation was as follows: wish to be dead (53.3\%); suicidal thoughts (47.2\%); suicidal thoughts with methods but without specific plan or intent to act (36.6\%); suicidal intent without specific plan (35.4\%); and suicidal intent with specific plan (1.8\%) (Table 2). The reported frequency distribution of suicidal ideation items was 1 time per week in $28.9 \%$ and $2-5$ times per week for $12.2 \%$ of cases. The duration of suicide ideation was fleeting, a few seconds or minutes for $21.9 \%$ and $<1$ hour or some of the time for $23.0 \%$. Regarding controllability of suicidal thoughts, $27.5 \%$ did not attempt to control them, while $33.9 \%$ and $10.4 \%$ of respondents could control then with little difficulty and with difficulty respectively. Deterrents, anyone or anything, did not apply to $27.6 \%$ of respondents as they only wished to be dead, while $24.2 \%$ agreed that deterrents probably stopped them from killing themselves. The mostly commonly endorsed reason for suicidal ideation was to get attention, revenge or a reaction from others or to end/stop pain (19.0\%).

\section{Associations}

No significant associations with items on the CSSRS were observed by age group ( $\leq 29$ and $\geq 30$ years). However, the demographic variables sex, family type, education and employment were all associated with items on the CSSRS (Tables 3 and 4).

Table 3 shows the significant demographic and other risk factors for attempted suicide. Among this group of depression sufferers, $46.3 \%$ of males 


\begin{tabular}{lcc}
\hline $\begin{array}{l}\text { Table 1 Activating events, treatment history and clinical status among the sample } \\
\text { of patients with depression }(\boldsymbol{n}=\mathbf{5 5 7})\end{array}$ & $\begin{array}{c}\text { No. of } \\
\text { respondents }\end{array}$ \\
\hline Variable & & \\
& 61 & 11.0 \\
Activating events & 221 & 39.7 \\
Recent loss or other significant negative event & & \\
Current or pending isolation or feeling alone & 282 & 50.6 \\
Treatment history & 279 & 50.1 \\
Previous psychiatric diagnosis \& treatments & 264 & 47.4 \\
Hopeless or dissatisfied with treatment & 118 & 21.2 \\
Noncompliant with treatment & & 70.9 \\
Not receiving treatment & 395 & 41.7 \\
Clinical status & 232 & 4.3 \\
Hopelessness & 24 & 53.0 \\
Highly impulsive behaviour & 295 & 3.9 \\
Substance abuse or dependence & 22 & 46.3 \\
Agitation or severe anxiety & 258 & 17.1 \\
Aggressive behaviour towards others & 95 & 37.5 \\
Perceived burden on family or others & 209 & 7.7 \\
Method of suicide available & 43 & \\
Refuses or unable to agree to safety plan & & \\
Family history of suicide & & \\
\hline
\end{tabular}

had made an actual suicide attempt compared with only $21.9 \%$ of females $(\mathrm{OR}=3.04 ; 95 \% \mathrm{CI}: 1.83-5.20)$. Those who were literate were significantly more likely to actually attempt suicide than those who were illiterate (49.7\% versus $19.5 \%)(O R=4.07 ; 95 \%$

CI: 2.53-6.55), so too were those in employment versus the unemployed ( $56.5 \%$ versus $32.5 \%)(\mathrm{OR}=2.70 ; 95 \%$ CI: 1.85-3.93). Respondents living in a joint family type were significantly much more likely to attempt suicide than those in a nuclear family $(65.2 \%$

\begin{tabular}{|c|c|c|}
\hline Variable & $\begin{array}{l}\text { No. of } \\
\text { respondents }\end{array}$ & $\%$ \\
\hline \multicolumn{3}{|l|}{ Suicide and self-injurious behaviours } \\
\hline Actual suicidal attempt & 204 & 36.6 \\
\hline Interrupted attempt & 166 & 29.8 \\
\hline Aborted or self-interrupted attempt & 193 & 34.6 \\
\hline Other preparatory acts to kill self & 105 & 18.9 \\
\hline $\begin{array}{l}\text { Self-injurious behaviour without suicide } \\
\text { intent }\end{array}$ & 43 & 7.7 \\
\hline \multicolumn{3}{|l|}{ Suicide ideation } \\
\hline Wish to be dead & 297 & 53.3 \\
\hline Suicidal thoughts & 263 & 47.2 \\
\hline $\begin{array}{l}\text { Suicidal thoughts with method but without } \\
\text { specific plan or intent to act }\end{array}$ & 204 & 36.6 \\
\hline Suicidal intent (without specific plan) & 197 & 35.4 \\
\hline Suicidal intent (with specific plan) & 10 & 1.8 \\
\hline
\end{tabular}

versus $17.6 \%)(\mathrm{OR}=8.75 ; 95 \% \mathrm{CI}$ : 5.75-13.3).

Other risk factors with significant associations were smoking and presence of physical comorbidity. Among the respondents, $59.1 \%$ of smokers had attempted suicide compared with only $24.5 \%$ of non-smokers ( $\mathrm{OR}=3.42 ; 95 \%$ CI: 2.34-4.98), while more than twothirds of those with physical comorbidity had made an actual suicide attempt (68.7\%) compared with only around one-third of those with no physical disorders $(32.7 \%)(\mathrm{OR}=4.41 ; 95 \% \mathrm{CI}$ : 2.81-6.90) (Table 3).

Table 3 shows similar associations for the other items of suicidal behaviour. Those who made an aborted suicide attempt or interrupted suicide were $>3$ times more likely to be male, $>8$ times more likely to live in a joint family, 3-4 times more likely to be literate and 2.4 times more likely to be employed (Table 3). Males and those living in a joint family were 10 times more likely to have made preparatory acts to kill themselves. Smoking and physical comorbidity were also significantly associated with aborted suicide attempt, interrupted attempt and other preparatory acts to kill self (Table 3 ).

Table 4 presents the significant demographic and other risk factors for the items of suicidal ideation. Male $\operatorname{sex}(\mathrm{OR}=1.67 ; 95 \% \mathrm{CI}: 1.08-2.59)$, joint family type $(\mathrm{OR}=11.4 ; 95 \% \mathrm{CI}$ : 7.55-17.2), literate educational level $(\mathrm{OR}=2.52$; 95\% CI: 1.70-3.74), being in employment $(\mathrm{OR}=2.45$; $95 \% \mathrm{CI}$ : 1.70-3.54), smoking ( $\mathrm{OR}=2.76$; $95 \%$ CI: 1.92-3.98) and having physical comorbidities (OR $=6.14$; 95\% CI: 3.74 10.1) were all significant risk factors for suicidal thoughts. Similar associations were seen for suicidal thoughts with method (but without a specific plan) or intent to act and suicidal intent without specific plan. Suicide intent without a specific plan was only associated with living in a joint family versus nuclear family ( $59.8 \%$ versus $17.3 \%$; $\mathrm{OR}=7.16$; 95\% CI: 4.74-10.8). 


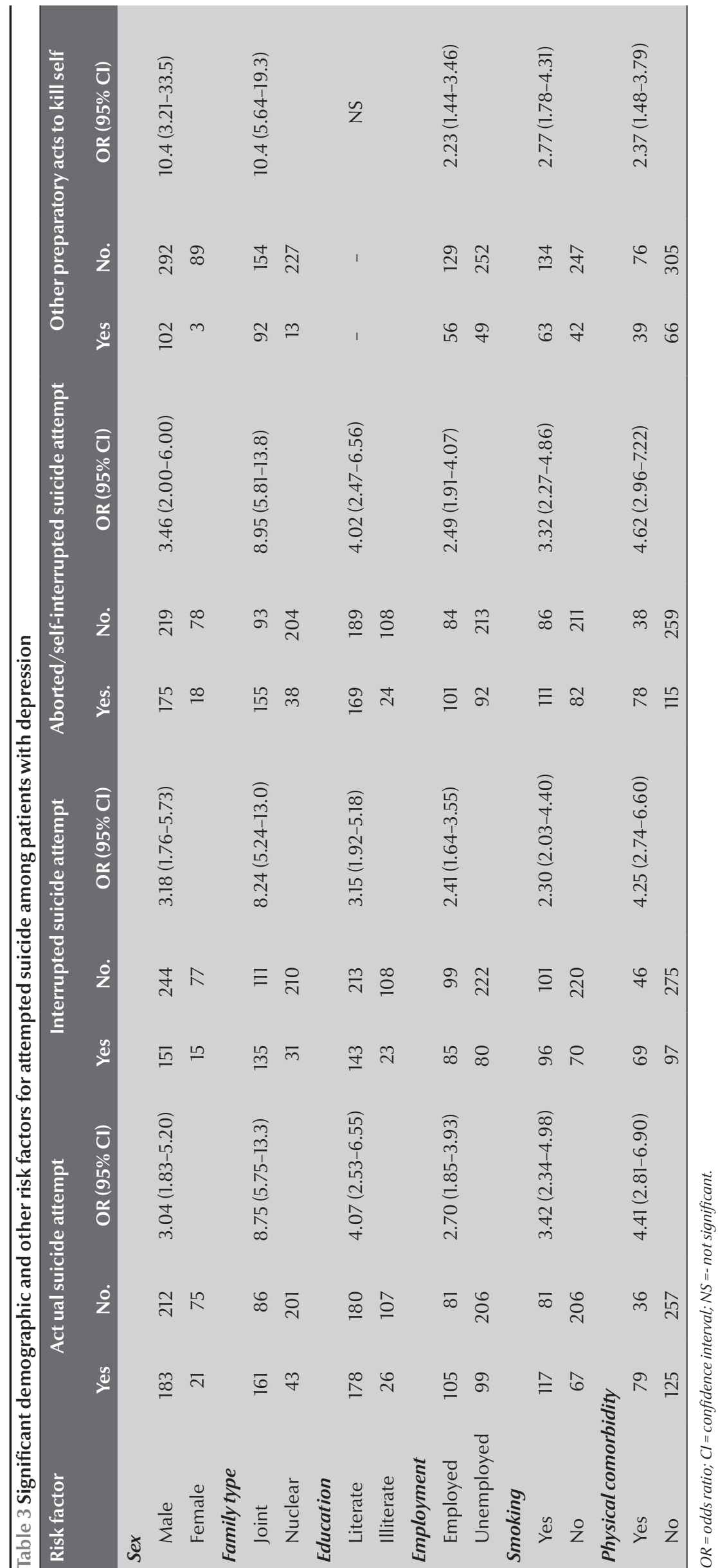

\section{Discussion}

According to this hospital-based study, a majority of patients with depression with or without suicidal behaviour were married adults having primary to college education with average income and threequarters of them were males. A little more than half of the patients were unemployed and living in joint families. Furthermore more than half of the patients were from an urban background and $60 \%$ were smokers. This sociodemographic profile of the studied population is consistent with other Asian studies [21]. It should be noted that in Saudi Arabia, all patients are eligible for treatment free of charge. From a clinical perspective, this study found that the majority of patients with depression were chronic smokers. Co-occurring physical diseases and anxiety and other mental disorders were found in $24.8 \%$ and $48.3 \%$ of patients respectively.

According to this hospital study, the prevalence of attempted suicide (36.6\%), suicidal thoughts $(47.2 \%)$ and preparatory acts to kill self (18.9\%) were higher than rates reported in community samples elsewhere. In a Chinese study, $17.4 \%$ of students had seriously considered attempting suicide and $8.1 \%$ had made a specific plan to attempt suicide during the 12 months preceding the survey [22]. In a Turkish study, 23\% of student participants reported having thoughts of killing themselves during the past 12 months or their life [23]. We would expect that the rate of suicidal and self-injurious behaviours in a psychiatric population would be higher than in the community, as shown in surveys of American youths [24]. The prevalence of suicidal and self-injurious behaviours was highly variable and attributed among others to a 


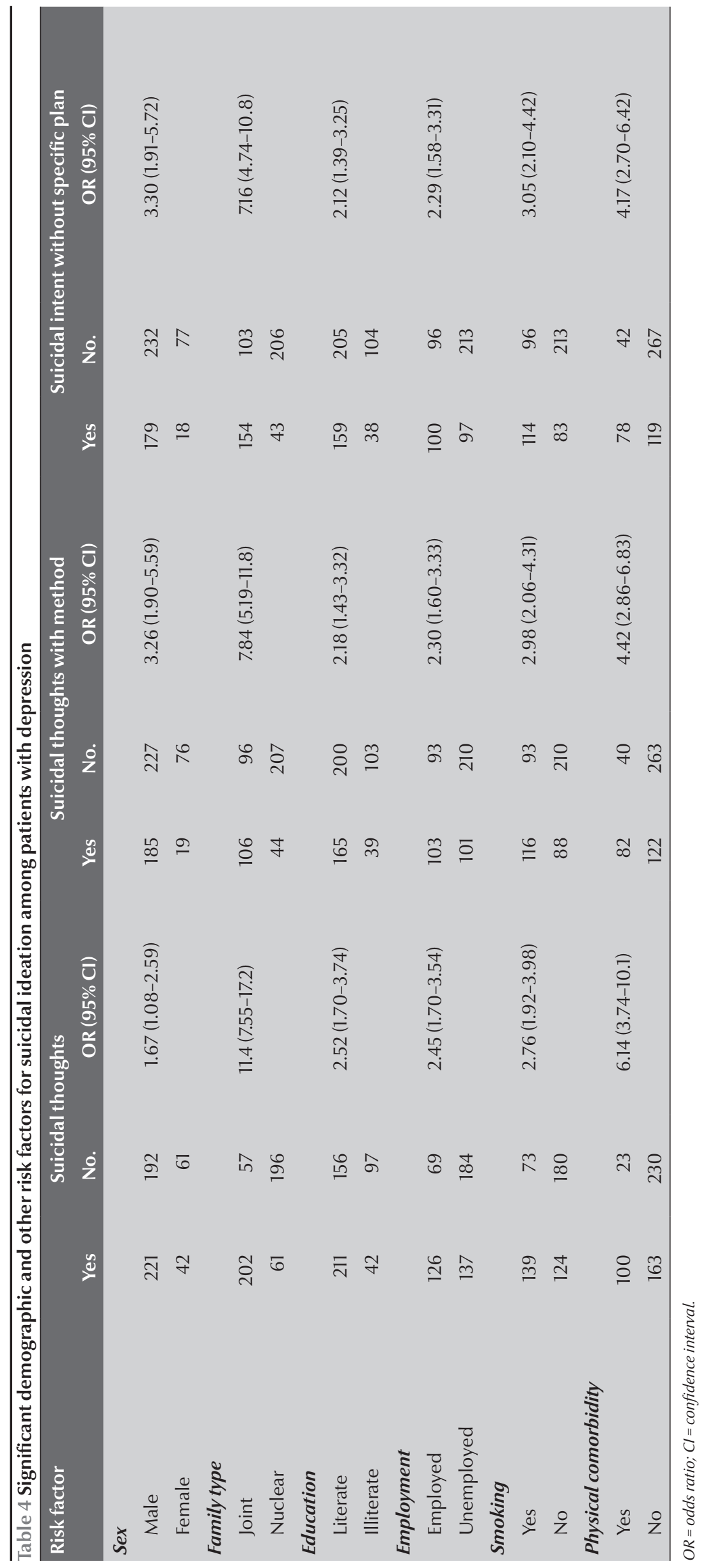

variety of factors including sex, age, study settings, sample characteristics and study design.

Protective factors against suicide and non-suicidal behaviours need to be highlighted. Several sociodemographic variables - sex, education, employment and family typewere found to have significant associations with attempted suicide and suicide ideation as revealed in other research $[9,11,12,25-28]$. By and large the world literature is replete with identified risk and protective factors of suicide and self-injurious behaviours including poverty, ethnicity, negative life events, stigma, previous attempts, impulsivity, drug abuse spirituality, social and family supports, job engagement and others $[26,27,29,30]$. In one study of adolescents, family connectedness as an indicator of joint families was found to be a strong protective factor against suicidal behaviour among adolescents with a history of sexual abuse [29]. In another study of the role of psychological buffers in the development of hopelessness and suicidal ideation, it was revealed that life satisfaction, self-esteem, perception of family cohesion and social supports were significant mitigating factors against hopelessness and suicidal ideation [30]. In a study of males farmers in Australia, sense of belonging to a spiritual community and increasing social support were important protective factors against suicidal ideation emerging from depression [16]. Detailed information on suicide and its risk and protective factors in terms of economic prosperity, poverty, unemployment, debts, rurality, urbanity, cultural, extended and joint families, and the role of women, nuclear families and religion is available from Asian countries [31].

This study has some caveats. It was a hospital-based research and therefore its results can not be 
generalized to the general population. The study found many significant associations but a multiple regression has yet to be carried out. The reliability and validity of the tool in the Saudi context was not assessed; however, the raters and researchers agreed on all probes of this questionnaire. In the light of these limitations, the results of this research should be interpreted with caution.
In summary, this cross-sectional, hospital-based study provided a broad idea about the prevalence of suicidal and non-suicidal self-injurious behaviours together with certain risk and protective factors among patients with depression and the data is comparable with international studies. Prospective community-based studies are needed in this prioritized research avenue in the future.

\section{Acknowledgements}

The authors express thanks to King Abdulaziz City for Science and Technology for financial support for this small grant research no. MS 12-17 and also to all the research participants and assistant researchers for their cooperation in completing this research.

\section{References}

1. Mental and neurological disorders. Fact Sheet No 265. Geneva, World Health Organization, 2001.

2. Peveler R, Carson A, Rodin G. Depression in medical patients. British Medical Journal, 2002, 325:149-152.

3. Harris EC, Barraclough B. Suicide as an outcome for mental disorders. A meta-analysis. British Journal of Psychiatry, 1997, 170:205-228.

4. Foster T, Gillespie K, McClelland R. Mental disorders and suicide in Northern Ireland. British Journal of Psychiatry, 1997, $170: 447-452$

5. Gunnell D et al. The epidemiology and management of selfharm amongst adults in England. Journal of Public Health, 2005, 27:1-5.

6. Sinclair J, Green J. Understanding resolution of deliberate self harm: qualitative interview study of patients' experiences. British Medical Journal, 2005, 330:1112-1115.

7. Shirodkar SS, Hammad FT, Qureshi NA. Male genital selfamputation in the Middle East. A simple repair by anterior urethrostomy. Saudi Medical Journal, 2007, 28:791-793.

8. Qureshi NA. Male genital self-mutilation: a case report with special emphasis on sociocultural meanings. Neurosciences (Riyadh), 2008, 13:445-449.

9. Mitchell AJ, Dennis M. Self harm and attempted suicide in adults: 10 practical questions and answers for emergency department staff. Emergency Medicine Journal, 2006, 23:251-255.

10. Pagura J et al.; Swampy Cree Suicide Prevention Team. Help seeking and perceived need for mental health care among individuals in Canada with suicidal behaviors. Psychiatric Services, 2009, 60:943-949.

11. Sokero TP et al. Prospective study of risk factors for attempted suicide among patients with DSM-IV major depressive disorder. British Journal of Psychiatry, 2005, 186:314-318.

12. Scoliers G et al. Sociodemographic and psychopathological risk factors for repetition of attempted suicide: a 5-year followup study. Archives of Suicide Research, 2009, 13:201-213.

13. Taliaferro LA et al. Spiritual well-being and suicidal ideation among college students. Journal of American College Health, 2009, 58:83-90

14. Lizardi D et al. Perceived reasons for living at index hospitalization and future suicide attempt. Journal of Nervous and Mental Disease, 2007, 195:451-455.

15. Fitzpatrick KM, Piko BF, Miller E. Suicide ideation and attempts among low-income African American adolescents. Suicide and Life-Threatening Behavior, 2008, 38:552-563.

16. McLaren S, Challis C. Resilience among men farmers: the protective roles of social support and sense of belonging in the depression-suicidal ideation relation. Death Studies, 2009, 33:262-276.

17. Taliaferro LA et al. High school youth and suicide risk: exploring protection afforded through physical activity and sport participation. Journal of School Health, 2008, 78:545-553.

18. Pettingell SL et al. Protecting urban American Indian young people from suicide. American Journal of Health Behavior, 2008, 32:465-476.

19. Khair OY, Al-Mudefer UI. [The motives of attempted suicide and the diagnosis of psychiatric disorders of persons who attempted suicide]. Arab Journal of Psychiatry, 2005, 16:161-172 [in Arabic].

20. Posner K et al. The Columbia-Suicide Severity Rating Scale: initial validity and internal consistency findings from three multisite studies with adolescents and adults. American Journal of Psychiatry, 2011, 168(12):1266-1277.

21. Hendin $\mathrm{H}$ et al., eds. Suicide and suicide prevention in Asia. Geneva, World Health Organization, 2008.

22. Cheng $\mathrm{Y}$ et al. Protective factors relating to decreased risks of adolescent suicidal behaviour. Child: Care, Health and Development, 2009, 35:313-322.

23. Eskin $\mathrm{M}$ et al. Risk factors for and protective factors against adolescent suicidal behavior in Turkey. Crisis, 2007, 28:131-139.

24. National Youth Risk Behavior Survey System. Trends in the prevalence of suicide-related behaviors. Altlanta, Georgia, Centers for Disease Control and Prevention, 1991-2007.

25. Hintikka J et al. Mental disorders in self-cutting adolescents. Journal of Adolescent Health, 2009, 44:464-467.

26. Rudd MD et al. Warning signs for suicide: theory, research, and clinical applications. Suicide and Life-Threatening Behavior, 2006, 36:255-262.

27. Wintersteen MB, Diamond GS, Fein JA. Screening for suicide risk in the pediatric emergency and acute care setting. Current Opinion in Pediatrics, 2007, 19:398-404.

28. O'Carroll PW et al. Beyond the Tower of Babel: a nomenclature for suicidology. Suicide and Life-Threatening Behavior, 1996, 26:237-252.

29. Eisenberg ME, Ackard DM, Resnick MD. Protective factors and suicide risk in adolescents with a history of sexual abuse. Journal of Pediatrics, 2007, 151:482-487.

30. Chioqueta AP, Stiles TC. The relationship between psychological buffers, hopelessness, and suicidal ideation: identification of protective factors. Crisis, 2007, 28:67-73.

31. Vijayakumar L et al. Socioeconomic, cultural and religious factors affecting suicide prevention in Asia. In: Hendin $\mathrm{H}$ et al., eds. Suicide and suicide prevention in Asia. Geneva, World Health Organization, 2008. 